\title{
Effect of Soft and Softer Handoffs on CDMA System Capacity
}

\author{
Chin-Chun Lee, Member, IEEE, and Raymond Steele, Fellow, IEEE
}

\begin{abstract}
The effect of soft and softer handoffs on codedivision multiple-access (CDMA) system capacity is evaluated for unsectorized and sectorized hexagonal cells according to an average bit energy-to-interference power spectral density, which corresponds to a bit-error rate (BER) of $10^{-3}$. The effect of imperfect sectorization on sectorization efficiency is also considered. On the reverse link, there is no capacity loss as no extra channels are needed to perform soft handoff, while the macrodiversity provided by soft handoff can improve the reverselink quality and extend the cell coverage. On the forward link, when soft handoff is employed in unsectorized cells, the capacity loss due to two traffic channels assigned to a user in the handoff zone is $0.2 \%$ or $1.1 \%$ for a voice activity factor of $3 / 8$ or $1 / 2$, respectively. As the forward-link capacity is higher than that of the reverse link, this small capacity loss does not affect the system capacity. For sectorized cells having three sectors per cell, there are overlapping coverage areas between sectors, where mobiles in these areas are subjected to an increase in cochannel interference. For an overlapping angle of $5^{\circ}$, the sectorization efficiency is 0.96 and 0.7 for the reverse-link and forward-link systems, respectively. When soft and softer handoffs are employed, the forward-link sectorization efficiency is improved to 0.97 . We find the application of soft and softer handoff improves not only the forward-link capacity, but also the signal-to-interference ratio (SIR) for mobiles near the cell and sector boundaries.
\end{abstract}

Index Terms-CDMA, macrodiversity, sectorization efficiency, soft handoff, softer handoff.

\section{INTRODUCTION}

$\mathbf{S}$ OFT HANDOFF in cellular code-division multiple-access (CDMA) systems is a technique whereby mobiles near cell boundaries communicate the same transmitted signals to more than one base station (BS) within their vicinity [1]-[3]. Soft handoff is important because it provides enhanced communication quality and a smoother transition compared to the conventional hard handoff. On the reverse-link, signals transmitted by mobiles in the handoff area may reach all the nearby BS's, even though the signals are not intended for them and the mobile signals appear as interference in these nearby cells. By putting more matched filters in the receiver, BS's can receive signals from mobiles in the nearby soft-handoff areas. Notice that no extra channels are required to accomplish

Manuscript received December 15, 1995; revised December 3, 1996.

C.-C. Lee was with the Department of Electrical Engineering, Chung Cheng Institute of Technology, Taoyuan, Taiwan, R.O.C. He is now with the Department of Communication and Computer Technology, Ta-Yeh University, Changhwa, Taiwan, R.O.C.

R. Steele is with the Department of Electronics and Computer Science, University of Southampton, Southampton, U.K.

Publisher Item Identifier S 0018-9545(98)05714-4. soft handoffs on the reverse links. Soft handoff provides macrodiversity, which is due to more than one BS being involved in the communications. The signal-to-interference ratio (SIR) is improved by combining the signals from the different BS's, and this, in turn, increases reverse-link quality and extends cell coverage [4], [5]. As there are at least two BS's involved in the soft-handoff process, where each BS supports a forward-link channel to the mobile, the number of available channels on the forward link decreases as the number of mobiles in soft handoff increases. We investigate this effect on the system capacity.

Sectorization in cellular CDMA systems increases the capacity in proportion to the number of sectors per cell. For a sectorized CDMA system, there are two kinds of handoff when mobiles move from one sector to another, namely, handoff between two sectors in different cells and between two sectors within a cell. Similar to soft handoff, softer handoff is the soft handoff between two sectors of the same cell [6]. For a perfect sectorized antenna pattern, softer handoff cannot be applied. In practice, the antenna patterns do not fit the sector area perfectly, and there is an overlapping of the two antenna patterns between the sectors [7]. The overlapping of the sector antenna patterns generates additional interference on both the reverse and forward links.

In this paper, the effect of soft and softer handoffs on the system capacity for unsectorized and sectorized cells is analyzed. The effect of imperfect sectorization is also considered. Our investigation was motivated by IS-95, which is the cellular CDMA mobile radio system standardized in the United States, as we could not find an in-depth analysis of the effect of soft and softer handoffs on the system capacity of IS-95, especially on the forward link, where two traffic channels are allocated to a mobile user. However, we will focus our analysis on a general cellular CDMA system rather than specifically for the IS-95 system. As we are concerned with the percentage of capacity change due to the application of soft and softer handoffs, we use in our analysis the average bit-energy-to-interference power spectral density (PSD) ratio instead of outage rate, which is the probability of bit-energyto-interference PSD being less than a required level.

CDMA is able to combat the effect of the fast fading if there are more than two multiple paths that can be resolved by the receiver [8]. By using a RAKE receiver incorporated with closed-loop power control, bit interleaving, channel coding, and space diversity reception, the effect of the fast fading can be reduced to a minimum [9], [10]. Therefore, a radio channel 


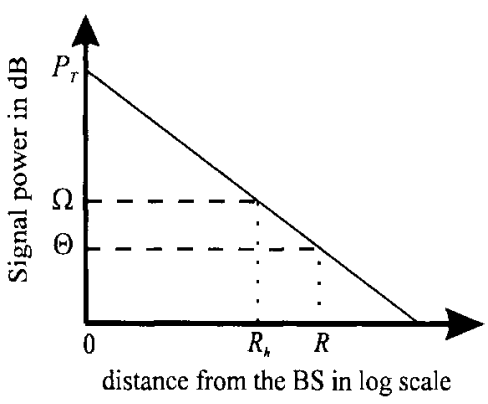

Fig. 1. Signal decays from the BS outwards. Only path loss is considered.

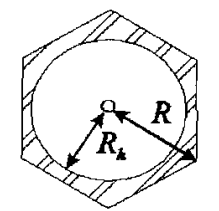

Fig. 2. Soft-handoff zone in hexagonal cells as shown shaded.

with only path loss and slow fading are considered in our analysis on the presumption that any fast fading would be effectively combated by the receiver.

\section{Soft Handoff IN UnSECTORIZED CELlS}

There is no extra channel needed to perform soft handoff on the reverse link, while the macrodiversity provided by soft handoff can improve the reverse-link quality by combining the signals from the BS's involved. As it is difficult to combine these signals at the mobile switching center (MSC), a selective combining scheme, which selects the bit frame having the minimal number of error bits, is more likely to be used [5]. Consequently, the link quality for mobiles in soft handoff is improved, or more users can be accommodated in exchange for the link-quality improvement. However, the capacity improvement depends upon the gain provided by the selective combining scheme. As there is no capacity loss on the reverse link, we will focus on the forward-link analysis hereafter.

On the forward link, the capacity loss due to the application of soft handoff in the unsectorized cells and the capacity gain due to the macrodiversity provided by the BS's involved in the soft-handoff process are investigated and compared. The effect of the soft handoff on the forward-link capacity in the unsectorized cells is then determined.

\section{A. Forward-Link Capacity Loss}

Consider a soft-handoff scheme, where soft handoffs involve $H$ BS's in unsectorized cells. In order to estimate the capacity loss due to soft handoff, we consider a radio channel whose path-loss exponent is $\alpha$ and ignore the shadow fading. As the mobiles travel from their BS to the boundary, the decrease of the received signal power level is shown in Fig. 1. The CDMA soft-handoff region in hexagonal cells with radius $R$ is between $R_{h}$ and $R$ from the $\mathrm{BS}$, as shown in Fig. 2 . When a mobile travels farther than the distance $R_{h}$ from its $\mathrm{BS}$, the soft-handoff process is initiated as the received signal level is less than a threshold $\Omega \mathrm{dB}$. The region between the distance from $R_{h}$ to $R$ is defined as the soft-handoff zone, and it has an area of

$$
\Psi_{h}=\frac{3 \sqrt{3}}{2} R^{2}-\pi R_{h}^{2}
$$

As soft handoff is achieved by the collaboration of $H(H \geq$ 2) neighboring BS's, the capacity corresponding to mobiles in a soft-handoff zone is reduced from $\rho \Psi_{h}$ to $\rho \Psi_{h} / H$, where $\rho$ is the user distribution density and is assumed to be uniform. The loss of capacity corresponding to the mobiles in this soft-handoff zone is

$$
\rho \Psi_{h}\left(1-\frac{1}{H}\right)=\frac{H-1}{H} \rho\left(\frac{3 \sqrt{3}}{2} R^{2}-\pi R_{h}^{2}\right)
$$

The fraction of capacity loss compared with the capacity without soft handoff is

$$
F_{h}=\frac{\Psi_{h}\left(1-\frac{1}{H}\right)}{3 \frac{\sqrt{3}}{2} R^{2}}=\frac{H-1}{H}\left(1-\frac{2 \pi}{3 \sqrt{3}} \frac{R_{h}^{2}}{R^{2}}\right) .
$$

We first of all select the handoff threshold $\Omega$ and the minimum detectable signal level for the mobile's receiver when it is located at the boundary $\Theta$ and assume that the transmitted power from a $\mathrm{BS}$ is $P_{T}$, then

$$
\Omega=10 \log \left(\frac{P_{T}}{R_{h}^{\alpha}}\right)
$$

and

$$
\Theta=10 \log \left(\frac{P_{T}}{R^{\alpha}}\right)
$$

The difference between the minimal detectable signal level $\Theta$ and the handoff threshold $\Omega$ is

$$
\Theta-\Omega=10 \log \left(\frac{P_{T} R^{-\alpha}}{P_{T} R_{h}^{-\alpha}}\right)=10 \alpha \log \left(\frac{R_{h}}{R}\right)
$$

and by rearranging (6)

$$
\frac{R_{h}}{R}=10^{(\Theta-\Omega) / 10 \alpha}
$$

Combining (3) into (7) gives the fraction of capacity loss as

$$
\begin{aligned}
F_{h} & =\frac{H-1}{H}\left(1-\frac{2 \pi}{3 \sqrt{3}} \frac{R_{h}^{2}}{R^{2}}\right) \\
& =\frac{H-1}{H}\left[1-\frac{2 \pi}{3 \sqrt{3}} 10^{(2(\Theta-\Omega) / 10 \alpha)}\right] .
\end{aligned}
$$

For a handoff threshold having $3 \mathrm{~dB}$ higher than the minimal detectable signal level [7] and a fourth path-loss law, the fraction of capacity loss can be calculated by (8). The capacity losses for a soft handoff with two and three BS's involved are $7.2 \%$ and $9.5 \%$, respectively. 


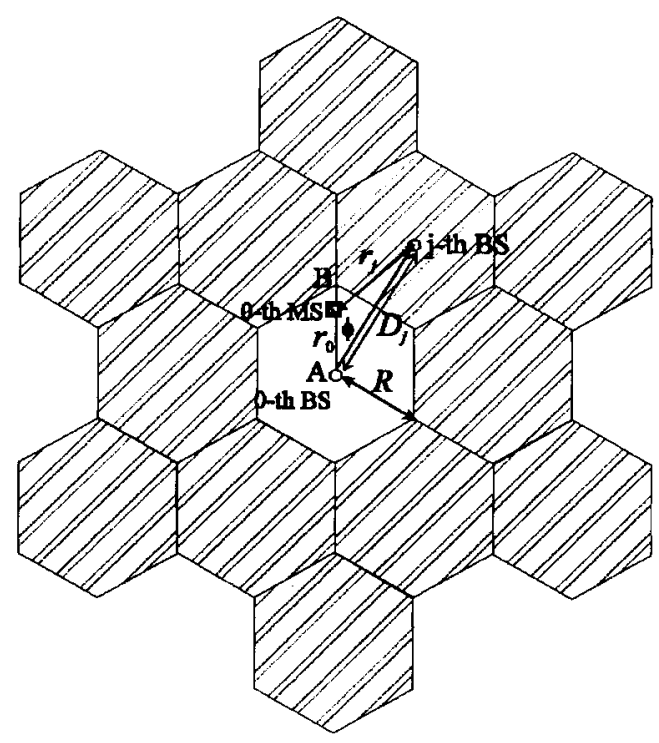

Fig. 3. Forward-link interference from the nearby 12 cochannel cells. Cochannel cells are shown shaded.

\section{B. Forward-Link Capacity Gain}

The main reason for employing soft handoff is that it provides macrodiversity by combining the signals transmitted by the involved $H$ BS's. As the probability that the signals from the $H$ BS's are all simultaneously subjected to deep shadowing is much smaller than that from a single BS, a gain in SIR can be obtained by combining the received signals from these $H$ BS's. This gain in SIR, in turn, increases the system capacity. By comparing the capacity with macrodiversity to that without macrodiversity, the gain of capacity of the CDMA forward-link system due to soft-handoff diversity can be determined.

1) Without Macrodiversity: Consider a CDMA forwardlink system with coherent demodulation, which is achieved by sending a CDMA pilot with all the traffic channels [9], [11]. Power control on the forward link is not critical as it is on the reverse link, and we will not include it in our deliberation. As mobiles near their BS's have a higher SIR than those mobiles near the boundary, the SIR on the forward link is dependent on the mobile's location. Consequently, the forward-link capacity is limited by the SIR when mobiles are located at the boundary.

Let us consider a mobile located in the zeroth cell at a distance of $r_{0}$ from the zeroth BS and at a distance of

$$
r_{j}=\sqrt{D_{j}^{2}+r_{0}^{2}-2 D_{j} r_{0} \cos (\phi)}
$$

from the $j$ th neighboring BS as shown in Fig. 3. In (9), $D_{j}$ is the distance between the zeroth and $j$ th BS's, and $\phi$ is defined in Fig. 3. For a radio channel with path-loss exponent $\alpha$ and a shadowing fading random variable $\lambda_{j}$ having a standard deviation of $\sigma$, the effect of path loss and shadowing between the zeroth mobile and zeroth BS is

$$
a_{0}^{2}=r_{0}^{-\alpha} 10^{\lambda_{0} / 10}
$$

while the effect of path loss and shadowing between the zeroth mobile and $j$ th $\mathrm{BS}$ is

$$
a_{j}^{2}=r_{j}^{-\alpha} 10^{\lambda_{j} / 10} \text {. }
$$

If each BS transmits a total power of $P_{T}$ to all its mobiles, then the zeroth mobile can receive a power of $P_{0}=a_{0}^{2} P_{T}$ from the zeroth BS and a power of $P_{j}=a_{j}^{2} P_{T}$ from the $j$ th BS. We assume that there are $N$ traffic channels and one pilot channel on the forward link, and each channel is allocated the same power. The signal power at the output of the matched filter is

$$
S=\frac{a_{0}^{2} P_{T}}{N+1}
$$

and the additive white Gaussian noise (AWGN)-to-signal ratio is

$$
\frac{\eta}{S}=\frac{N_{0} W}{S}
$$

where $N_{0}$ is the single-sided power spectral density of AWGN and $W$ is the message bandwidth. The intracellular interference-to-signal ratio from the other channels in the same cell is

$$
\frac{I_{\text {int }}}{S}=\frac{1}{S G_{p}} \sum_{i=1}^{N} v_{i} \frac{a_{0}^{2} P_{T}}{(N+1)}=\frac{1}{G_{p}} \sum_{i=1}^{N} v_{i}
$$

and the intercellular interference-to-signal ratio from the external $J-1$ surrounding BS's is

$$
\begin{aligned}
\frac{I_{\mathrm{ext}}}{S} & =\frac{1}{S} \frac{1}{2 G_{p}} \sum_{j=1}^{J-1} \sum_{i=0}^{N} v_{i j} a_{j}^{2} \frac{P_{T}}{N+1} \\
& =\frac{1}{2 G_{p}} \sum_{j=1}^{J-1} \sum_{i=0}^{N} v_{i j} \frac{a_{j}^{2}}{a_{0}^{2}}
\end{aligned}
$$

where the subscripts int and ext represent the interference from within the cell and external to the cell, respectively, $G_{p}$ is the processing gain, and $v_{i}$ as well as $v_{i j}$ represent the voice activity variables. The voice activity variable is binomial distributed and is equal to one with probability of $\mu$ and to zero with probability of $1-\mu$, where $\mu$ is defined as the voice activity factor (VAF). In (15), the intercellular interference-to-signal ratio is reduced by factor of two due to the carrier incoherence. From (10) and (11), $a_{j}^{2} / a_{0}^{2}$ in (15) can be rewritten as

$$
\frac{a_{j}^{2}}{a_{0}^{2}}=\left(\frac{r_{0}}{r_{j}}\right)^{\alpha} 10^{\left(\lambda_{j}-\lambda_{0}\right) / 10}=\left(\frac{r_{0}}{r_{j}}\right)^{\alpha} 10^{\zeta_{j} / 10}
$$

where $\zeta_{j}=\lambda_{j}-\lambda_{0}$ is a normal random variable having a standard deviation of $\sqrt{2} \sigma$ as $\lambda_{0}$ and $\lambda_{j}$ are two independent fading variables. Substituting (16) into (15) yields

$$
\frac{I_{\mathrm{ext}}}{S}=\frac{1}{2 G_{p}} \sum_{j=1}^{J-1} \sum_{i=0}^{N} v_{i j}\left(\frac{r_{0}}{r_{j}}\right)^{\alpha} 10^{\zeta_{j} / 10} .
$$

Unlike the intracellular interference-to-signal ratio, the intercellular interference-to-signal ratio depends on the mobile's location and the shadowing condition. 
From (13), (14), and (17), the bit energy-to-interference power spectral density ratio (PSD) ratio $E_{b} / I_{0}$ at the output of the matched filter is

$$
\frac{E_{b}}{I_{0}}=\frac{S}{I}=\frac{1}{\frac{I_{\mathrm{int}}}{S}+\frac{I_{\mathrm{ext}}}{S}+\frac{\eta}{S}} .
$$

From (18), $E_{b} / I_{0}$ is a convex function of $\left(I_{\mathrm{int}}+I_{\mathrm{ext}}\right) / S$. From Jensen's inequality, we have

$$
E\left[\frac{E_{b}}{I_{0}}\right] \geq \frac{1}{E\left[\frac{I_{\mathrm{int}}}{S}+\frac{I_{\mathrm{ext}}}{S}\right]+\frac{\eta}{S}} .
$$

Substituting (14) and (17) into (19) yields (20), given at the bottom of the page, where

$$
\phi_{j}\left(\zeta_{j}, \frac{r_{0}}{r_{j}}\right)= \begin{cases}1, & \text { for }\left(\frac{r_{0}}{r_{j}}\right)^{\alpha} 10^{\zeta_{j} / 10} \leq 1 \\ 0, & \text { otherwise }\end{cases}
$$

is a constraint function, which accounts for the mobiles at the boundaries tending to communicate to a BS that offers the least signal attenuation under fading conditions. From [9], the mean in the denominator of (20) can be shown to be

$$
\begin{aligned}
E\left[\phi_{j}\left(\zeta_{j}, \frac{r_{0}}{r_{j}}\right) 10^{\zeta_{j} / 10}\right] & \\
= & \exp \left(\frac{\sigma \ln (10)}{10}\right)^{2} \\
& \cdot\left\{1-Q\left[\frac{10 \alpha \log \left(\frac{r_{j}}{r_{0}}\right)}{\sqrt{2} \sigma}-\frac{\sqrt{2} \ln (10) \sigma}{10}\right]\right\} .
\end{aligned}
$$

The forward-link performance in terms of bit-error rate (BER) is given by

$$
\mathrm{BER}=\frac{1}{2} \operatorname{erfc}\left(\sqrt{E\left[\frac{E_{b}}{I_{0}}\right]}\right)
$$

where $\operatorname{erfc}(\bullet)$ is the complementary error function. For a BER less than $10^{-3}$, the required average bit energy-to-interference PSD ratio $\gamma_{\text {req }}=\left(E_{b} / I_{0}\right)_{\text {req }}$ is $7 \mathrm{~dB}$. According to this specific $\gamma_{\text {req }}$, the forward-link capacity can be evaluated from (20), namely,

$$
N \geq \frac{1+\frac{G_{p}}{\mu}\left(\frac{1}{\gamma_{\mathrm{req}}}-\frac{\eta}{S}\right)}{1+\frac{1}{2} \sum_{j=1}^{J}\left(\frac{r_{0}}{r_{j}}\right)^{\alpha} E\left[\phi_{j}\left(\zeta_{j}, \frac{r_{0}}{r_{j}}\right) 10 \zeta_{j} / 10\right]}-1 .
$$

For a CDMA system having a processing gain of $21 \mathrm{~dB}$ (127 chips per information bit) and an $S / \eta$ of $20 \mathrm{~dB}$, the

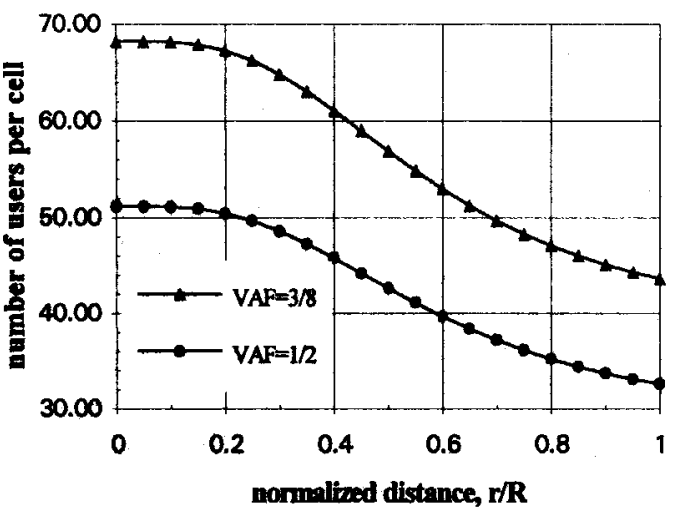

Fig. 4. Forward-link capacity without soft-handoff diversity as a function of the distance from the cell site toward the cell boundary.

forward-link capacity in terms of the minimal number of users per cell for different values of normalized distance $r_{0} / R$ can be calculated from (24). By considering the 12 cochannel interfering cells shown in Fig. 3, the curves of the capacity of the forward-link system along line $\mathrm{AB}$ in Fig. 3 are shown in Fig. 4 for a VAF of $3 / 8$ and $1 / 2$. According to the capacity variation, the capacity for mobiles near its BS is higher than that for mobiles at the boundaries. In order for a BS to serve all the mobiles in its cell with a minimal quality requirement, the system capacity tends to be limited by mobiles at the boundaries. From the Fig. 4, the capacity at the boundary without macrodiversity is 32 and 43 users per cell for VAF's of $1 / 2$ and $3 / 8$, respectively.

2) With Macrodiversity: The SIR of the mobiles within the soft-handoff zone can be improved by combining the received signals from the $H$ BS's. Therefore, the capacity can be increased in proportion to the increase in SIR. As shown in Fig. 5, the mobile receiver has $H$-matched filters to detect the $H$ signals coming from the $H$ neighboring BS's. After demodulating with the carrier, which is provided by the pilot from its own BS, the $H$-received signals are matched with their corresponding spreading codes. The outputs of the $H$-matched filters are cophased and combined.

The channel attenuation can be estimated from the CDMA pilot signal, and maximal ratio combining is performed by weighting the amplitudes of the $H$ signals according to their channel attenuation [12], [13]. The $H$-weighted signal amplitudes are cophased and summed together to give a maximal amplitude, where polarity determines the logical value of the regenerated bit. The combined $E_{b} / I_{0}$ at the output of the maximal ratio combiner can be shown to be [13]

$$
\left(\frac{E_{b}}{I_{0}}\right)_{c}=\sum_{i=0}^{H-1}\left[\frac{E_{b}}{I_{0}}\right]_{i}
$$

$$
E\left[\frac{E_{b}}{I_{0}}\right] \geq \frac{1}{\frac{\mu N}{G_{p}}+\frac{\mu(N+1)}{2 G_{p}} \sum_{j=1}^{J-1}\left(\frac{r_{0}}{r_{j}}\right)^{\alpha} E\left[\phi_{j}\left(\zeta_{j}, \frac{r_{0}}{r_{j}}\right) 10 \zeta_{j} / 10\right]+\frac{\eta}{S}}
$$




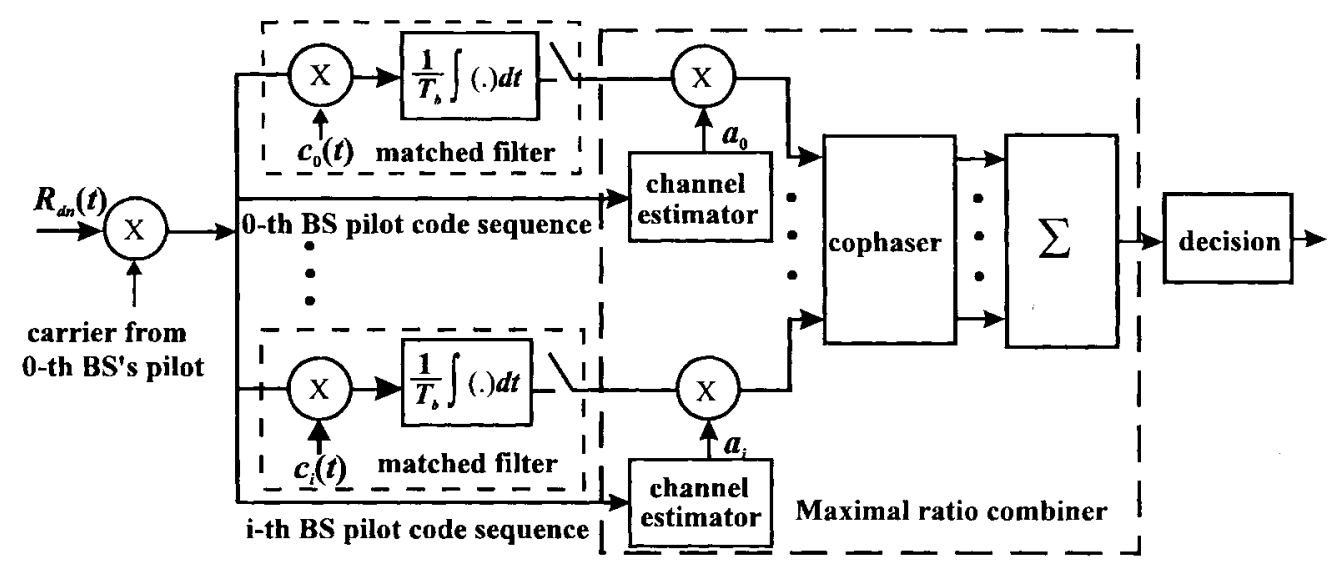

Fig. 5. Mobile receiver block diagram.

where $\left[E_{b} / I_{0}\right]_{i}$ is the bit energy-to-interference power spectral density ratio of the received signal from the $i$ th $\mathrm{BS}$, which is given in (19). From Fig. 5, we notice that only the traffic signal transmitted from zeroth BS can be demodulated coherently as its demodulating carrier is provided by the pilot signal from zeroth BS.

For a special case of soft handoff with two BS's involved, i.e., $H=2$, (25) becomes

$$
E\left[\frac{E_{b}}{I_{0}}\right]_{c}=E\left[\frac{E_{b}}{I_{0}}\right]_{0}+E\left[\frac{E_{b}}{I_{0}}\right]_{1}
$$

where $E\left[E_{b} / I_{0}\right]_{0}$ is given in (20) and $E\left[E_{b} / I_{0}\right]_{1}$ is shown in (27), given at the bottom of the page. In (27), the term $2\left(r_{1} / r_{0}\right)^{\alpha}$ in the denominator is the interference from the zeroth cell, where the factor two is due to the coherence of the demodulation carrier with the carrier from zeroth cell BS. For mobiles in the soft-handoff zone, the cochannel interference dominates the AWGN component in the $E_{b} / I_{0}$. By neglecting $\eta / S$ in (20) and (27) and substituting these equations into (26) yields (28), given at the bottom of the page. For a given $\gamma_{\text {req, }}$, the number of users per cell with handoff diversity can be obtained from (28) as (29), given at the bottom of the page.

For a CDMA forward-link system having a processing gain of $21 \mathrm{~dB}$ (127 chips per information bit) and an $S / \eta$ of 20 $\mathrm{dB}$, the capacity in terms of the minimal number of users per cell for different values of normalized $r_{0} / R$ in the softhandoff zone can be calculated from (29). By considering the

$$
\begin{aligned}
& E\left[\frac{E_{b}}{I_{0}}\right]_{1} \geq \frac{1}{\frac{\mu N}{G_{p}}+\frac{\mu(N+1)}{G_{p}}\left[2\left(\frac{r_{1}}{r_{0}}\right)^{\alpha}+\sum_{j=2}^{J-1}\left(\frac{r_{1}}{r_{j}}\right)^{\alpha}\right] E\left[\phi_{j}\left(\zeta_{j}, \frac{r_{0}}{r_{j}}\right) 10 \zeta_{j} / 10\right]+\frac{\eta}{S}} \\
& E\left[\frac{E_{b}}{I_{0}}\right]_{c}=E\left[\frac{E_{b}}{I_{0}}\right]_{0}+E\left[\frac{E_{b}}{I_{0}}\right]_{1} \\
& \geq \frac{\frac{G_{p}}{\mu N}\left\{2+\frac{1}{2} \sum_{j=1}^{J-1}\left(\frac{r_{0}}{r_{j}}\right)^{\alpha} E\left[\phi_{j}\left(\zeta_{j}, \frac{r_{0}}{r_{j}}\right) 10^{\zeta_{j} / 10}\right]+\left[2\left(\frac{r_{1}}{r_{0}}\right)^{\alpha}+\sum_{j=2}^{J-1}\left(\frac{r_{1}}{r_{j}}\right)^{\alpha}\right] E\left[\phi_{j}\left(\zeta_{j}, \frac{r_{0}}{r_{j}}\right) 10^{\zeta_{j} / 10}\right]\right\}}{\left\{1+\frac{1}{2} \sum_{j=1}^{J-1}\left(\frac{r_{0}}{r_{j}}\right)^{\alpha} E\left[\phi_{j}\left(\zeta_{j}, \frac{r_{0}}{r_{j}}\right) 10^{\zeta_{j} / 10}\right]\right\}\left\{1+\left[2\left(\frac{r_{1}}{r_{0}}\right)^{\alpha}+\sum_{j=2}^{J-1}\left(\frac{r_{1}}{r_{j}}\right)^{\alpha}\right] E\left[\phi_{j}\left(\zeta_{j}, \frac{r_{0}}{r_{j}}\right) 10^{\zeta_{j} / 10}\right]\right\}} \\
& N \geq \frac{\frac{G_{p}}{\mu \gamma_{\mathrm{req}}}\left\{2+\frac{1}{2} \sum_{j=1}^{J-1}\left(\frac{r_{0}}{r_{j}}\right)^{\alpha} E\left[\phi_{j}\left(\zeta_{j}, \frac{r_{0}}{r_{j}}\right) 10^{\zeta_{j} / 10}\right]+\left[2\left(\frac{r_{1}}{r_{0}}\right)^{\alpha}+\sum_{j=2}^{J-1}\left(\frac{r_{1}}{r_{j}}\right)^{\alpha}\right] E\left[\phi_{j}\left(\zeta_{j}, \frac{r_{0}}{r_{j}}\right) 10^{\zeta_{j} / 10}\right]\right\}}{\left\{1+\frac{1}{2} \sum_{j=1}^{J-1}\left(\frac{r_{0}}{r_{j}}\right)^{\alpha} E\left[\phi_{j}\left(\zeta_{j}, \frac{r_{0}}{r_{j}}\right) 10^{\zeta_{j} / 10}\right]\right\}\left\{1+\left[2\left(\frac{r_{1}}{r_{0}}\right)^{\alpha}+\sum_{j=2}^{J-1}\left(\frac{r_{1}}{r_{j}}\right)^{\alpha}\right] E\left[\phi_{j}\left(\zeta_{j}, \frac{r_{0}}{r_{j}}\right) 10^{\zeta_{j} / 10}\right]\right\}}
\end{aligned}
$$




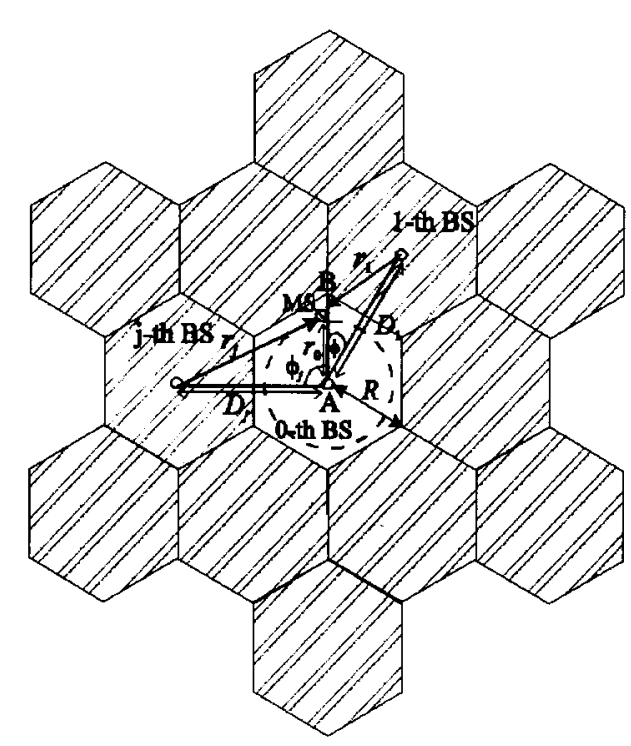

Fig. 6. Interference to mobiles in the soft-handoff zone from the nearby 12 cochannel cells. Cochannel cells are shown shaded.

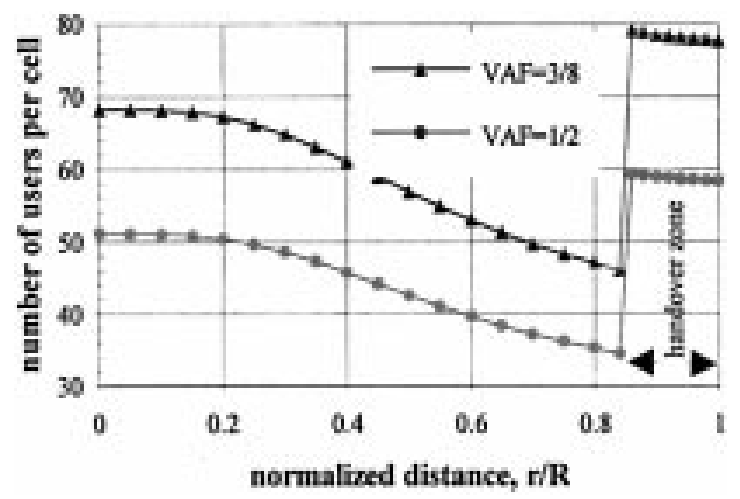

Fig. 7. Forward-link capacity with macrodiversity for mobiles in the soft-handoff zone.

12 cochannel interfering cells shown in Fig. 6, the curves of the capacity of the forward-link system for a VAF of $3 / 8$ and $1 / 2$ are shown in Fig. 7. Notice that the capacity for mobiles in the soft-handoff zone is much higher than that for mobiles in nonhandoff zone, hence, the capacity becomes limited by mobiles at $r=0.84 R$. The capacity gain on the forward link is the capacity difference between the capacity for mobiles at $r=R$ and $R_{h}=0.84 R$. From Fig. 4 , the capacity for mobiles at $r=R$ is 43 and 32 users per cell for a VAF of $3 / 8$ and $1 / 2$, respectively, while the capacity for mobiles at $R_{h}=0.84 R$ is 46 and 34 users per cell for VAF's of $3 / 8$ and $1 / 2$, respectively. Consequently, the capacity gain due to macrodiversity is three and two users per cell, which corresponds to a gain of $7.0 \%$ and $6.1 \%$ for VAF's of $3 / 8$ and $1 / 2$, respectively. As the system capacity is limited by $R_{h}=0.84 R$, the excess capacity due to macrodiversity becomes an increase in SIR for mobiles in soft handoff.

For a soft handoff with two BS's, the loss in the number of users per cell due to two traffic channels assigned to a user is $7.2 \%$, but the gain in the number of users per cell due to macrodiversity is $7.0 \%$ or $6.1 \%$ for VAF's of $3 / 8$ or $1 / 2$, respectively. The overall forward-link system losses are $0.2 \%$

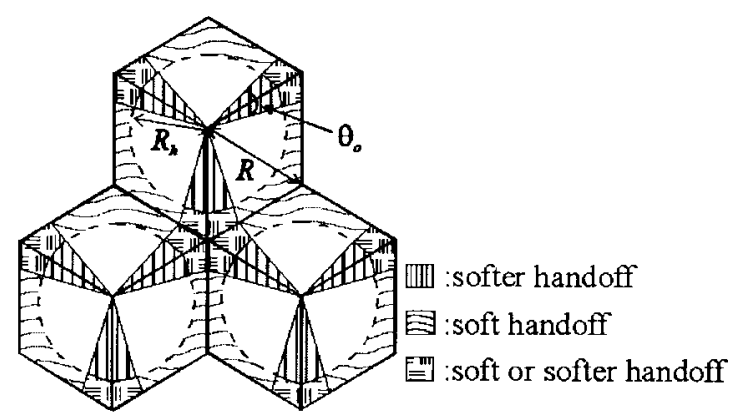

Fig. 8. Soft, softer, and soft or softer handoff subzones in sectorized cells.

and $1.1 \%$ of the number of users per cell due to the softhandoff process for VAF's of $3 / 8$ and $1 / 2$, respectively. The forward-link capacity is higher than the reverse-link capacity due to coherent demodulation, so the application of soft handoff does not have much effect on the system capacity. On the other hand, the communication quality for mobiles at the boundary can be improved by macrodiversity.

\section{Soft And Softer Handoff In Sectorized Cells}

Sectorization is used in CDMA to increase system capacity, where cells are divided into sectors and the same frequency spectrum is reused in every sector. The capacity is multiplied according to the number of sectors introduced. We limit the sectorization to three sectors per cell for our investigation, although other arrangements, e.g., four or six sectors per cell, are also used in practice. There are two kinds of handoffs in the sectorized CDMA system, namely, handoff between two sectors in different cells and handoff between two neighboring sectors in the same cell. The former one is exactly the same as the soft handoff we discussed in Section II, while the latter is defined as softer handoff. As softer handoff is handled by two BS's at the same cell site, there is a capacity loss. On the other hand, the macrodiversity of the two BS's can improve the SIR, and thereby the capacity increases proportionately. In this section, the effect of system capacity on both reverse and forward links are examined.

\section{A. Forward-Link Capacity Loss}

For a perfect sectorized antenna pattern, softer handoff cannot be applied. Antennas whose patterns form overlapping zone at sector boundaries are therefore used. We will consider the overlapping softer handoff coverage areas shown in Fig. 8. We model these areas as segments of a cell with angle $\theta_{o}$ between the sector borders. As shown in Fig. 8, the handoff zones in sectorized cells can be categorized into soft, softer, and soft or softer handoff subzones, where the soft or softer handoff subzone is an overlapping area between the soft and softer handoff subzones. Mobiles located in the soft or softer handoff subzone may be handled by either soft or softer handoff. Consequently, only mobiles located in the softer handoff subzone must be handled by softer handoffs. The softer handoff subzone has an area of

$$
\Psi_{s h}=\frac{1}{2} \theta_{o} R_{h}^{2}
$$




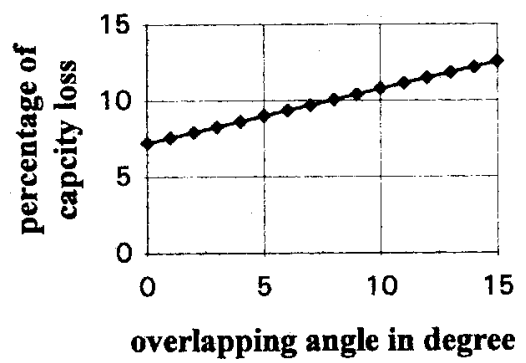

Fig. 9. Fraction of the forward-link capacity loss due to soft and softer handoffs. $H=2$.

We assume that the users are uniformly distributed with a density of $\rho$. Because the mobiles in the softer handoff zones may be handled by two BS's, the loss of capacity due to the mobiles in these zones is $1 / 2 \rho \Psi_{s h}$. The fraction of the capacity loss due to softer handoffs is

$$
F_{s h}=\frac{\frac{1}{2} \Psi_{s h}}{\frac{\sqrt{3}}{2} R^{2}}=\frac{\theta_{o}}{4 \sqrt{3}}\left(\frac{R_{h}}{R}\right)^{2}
$$

From Fig. 8, the total fractional capacity loss due to soft and softer handoffs in a sector is

$$
\begin{aligned}
F_{T} & =\frac{1}{2}\left[\frac{\left(\frac{\sqrt{3}}{2} R^{2}-\frac{1}{2} \frac{2 \pi}{3} \pi R_{h}^{2}\right)+\frac{1}{2} \theta_{o} R_{h}^{2}}{\frac{\sqrt{3}}{2} R^{2}}\right] \\
& =\frac{1}{2}\left[1-\frac{1}{\sqrt{3}}\left(\frac{2 \pi}{3}-\theta_{\circ}\right)\left(\frac{R_{h}}{R}\right)^{2}\right]
\end{aligned}
$$

where $R_{h}$ is the distance where the soft handoff starts. Notice that $F_{T}$ in (32) is also a sum of $F_{h}$ and $F_{s h}$ in (3) and (31), respectively.

The fraction of the capacity loss due to soft and softer handoffs in a sector as a function of overlapping angle $\theta_{o}$ is shown in Fig. 9. For a $\theta_{\circ}$ of $5^{\circ}$ and a handoff threshold that is 3 $\mathrm{dB}$ higher than the minimal detectable signal level, the capacity loss due to soft and softer handoffs is $9 \%$. Compared to the capacity loss due to soft handoffs in unsectorized cells, there is an additional capacity loss of $1.8 \%$ due to softer handoffs.

\section{B. Forward-Link Capacity Gain}

The capacity gain due to the macrodiversity on the forward link is evaluated in this section. We commence by calculating

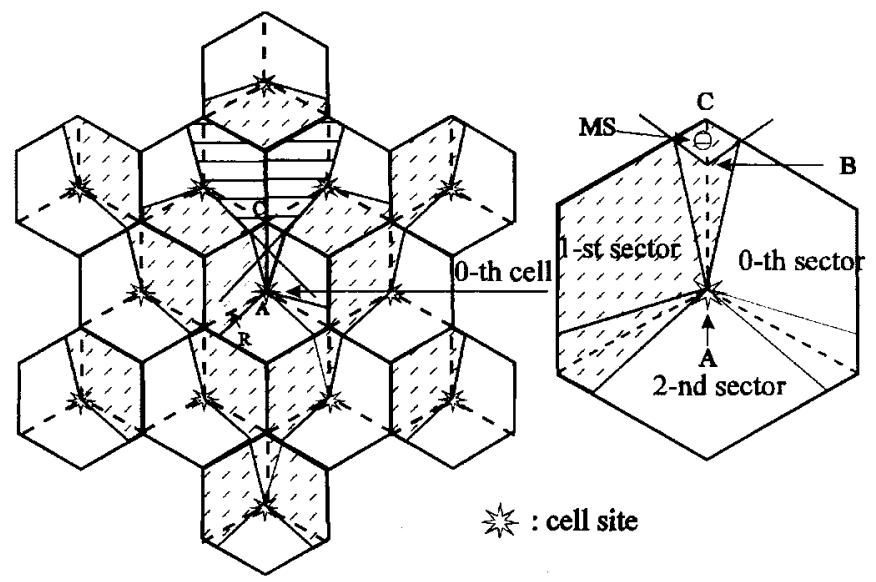

Fig. 10. Interference to mobiles in the antenna overlapping area from BS's in the surrounding cells. The interfering sectors are shown shaded.

the forward-link capacity without macrodiversity and then with macrodiversity. The results are then compared. From Section II, we find that on the forward link, the capacity is limited by the mobiles located at the cell boundaries, where the intercellular interference was higher than for mobiles inside the cell. We consider the areas having the highest interference level in evaluating the capacity.

1) Without Macrodiversity: We commence by considering the zeroth sector of the zeroth cell, where the significant interfering sectors are shown shaded in Fig. 10. Notice that in addition to the 13 surrounding interfering sectors, the first sector is also an interfering sector for mobiles in the antenna overlapping areas. For mobiles located near the corner of the hexagon, between $\mathrm{B}$ and $\mathrm{C}$, there are two more interfering sectors in Fig. 10 shown by the horizontal lines shaded pattern compared with the number of interfering sectors in the perfect sector antenna pattern arrangement.

As the zeroth BS and the first BS are at the same cell site forming the zeroth and first sectors, respectively, the path loss and shadowing conditions from both BS's to the mobile in the softer handoff zone are similar. For simplicity, we assume that the received power from the first sector BS and from the zeroth sector BS are the same, i.e., $\left(r_{0} / r_{1}\right)^{\alpha} 10^{\lambda_{1}-\lambda_{0}}=1$. From (19), without macrodiversity the $E_{b} / I_{0}$ for mobiles in this overlapping area is shown in (33), given at the bottom of the page, where the second term in the denominator is the interference power from the first sector. For a BER less than $10^{-3}, \gamma_{\text {req }}$ is $7 \mathrm{~dB}$. According to this specific $\gamma_{\text {req }}$, the

$$
\begin{aligned}
E\left[\frac{E_{b}}{I_{0}}\right] & \geq \frac{1}{\frac{\mu N}{G_{p}}+\frac{\mu(N+1)}{2 G_{p}}\left(\frac{r_{0}}{r_{1}}\right)^{\alpha} E\left[10^{\left(\lambda_{1}-\lambda_{0}\right) / 10}\right]+\frac{\mu(N+1)}{2 G_{p}} \sum_{j=2}^{J-1}\left(\frac{r_{0}}{r_{j}}\right)^{\alpha} E\left[\phi_{j}\left(\zeta_{j}, \frac{r_{0}}{r_{j}}\right) 10^{\zeta_{j} / 10}\right]+\frac{\eta}{S}} \\
& \cong \frac{1}{\frac{\mu N}{G_{p}}+\frac{\mu(N+1)}{2 G_{p}}+\frac{\mu(N+1)}{2 G_{p}} \sum_{j=2}^{J-1}\left(\frac{r_{0}}{r_{j}}\right)^{\alpha} E\left[\phi_{j}\left(\zeta_{j}, \frac{r_{0}}{r_{j}}\right) 10 \zeta_{j} / 10\right]+\frac{\eta}{S}}
\end{aligned}
$$


forward-link capacity can be evaluated from (33) as

$$
N \geq \frac{1+\frac{G_{p}}{\mu}\left(\frac{1}{\gamma_{\text {req }}}-\frac{\eta}{S}\right)}{1.5+\frac{1}{2} \sum_{j=1}^{J-1}\left(\frac{r_{0}}{r_{j}}\right)^{\alpha} E\left[\phi_{j}\left(\zeta_{j}, \frac{r_{0}}{r_{j}}\right) 10 \zeta_{j} / 10\right]}-1 .
$$

Next, we examine the situation when mobiles are located in the central part of a sector rather than in an area with overlapping sectors. The minimal number of users per sector can be calculated from (24) according to the interfering sectors shown in Fig. 11. From the figure, when mobiles are located near the boundary, between $\mathrm{D}$ and $\mathrm{E}$, there are two more interfering sectors compared to other locations in the nonsofter handoff zone. Consequently, the minimal number of users per sector that can be supported by the system occurs in the small area from $\mathrm{D}$ to $\mathrm{E}$, especially at E. For an overlapping angle less than $8^{\circ}$, location $\mathrm{D}$ is about $0.14 R$ from location $\mathrm{E}$, or $0.86 R$ from its BS. As this area (between D and E) is in the softhandoff zone, the additional interference due to overlapping sector antenna pattern can be combatted by the macrodiversity provided by the soft-handoff process.

For a CDMA forward-link system having a processing gain of $21 \mathrm{~dB}$ (127 chips), an $S / \eta$ of $20 \mathrm{~dB}$ and an overlapping angle of $5^{\circ}$, the minimal number of users per sector for different distances from the BS to the cell boundary, may be calculated from (24) and (34) for mobiles located in the central part of a sector and in the areas where sectors overlap, respectively. The graphs in terms of number of users per sector for the forwardlink system along the lines AE (in the central part of a sector) and AC (in the antenna overlapping areas) as a function of the normalized distance $r / R$ are given in Fig. 12. Notice that there is a sudden drop in the curves near $r / R=0.9$ because of the additional interference created by the overlapping antenna patterns. The worst SIR occurs when the mobiles are located at the soft or softer handoff subzones, especially at the corner $\mathrm{C}$, where the forward-link system can support 22 and 30 users per sector for VAF's of $1 / 2$ and $3 / 8$, respectively. Therefore, in sectorized cells, the forward-link capacity without soft and softer handoffs is limited by this minimal number of users per sector in the soft or softer handoff subzones.

2) With Macrodiversity: As the worst forward-link SIR occurs when mobiles are located in the area where the BS sectorized antenna patterns overlap, we will evaluate the capacity gain due to macrodiversity for mobiles located in these areas. Because the mobiles in soft or softer handoffs are able to employ maximal ratio combining of the two signals from the two neighboring sector BS's, the combined $E_{b} / I_{0}$ can be calculated by following a similar procedure to the one employed in Section II-B.

$$
\begin{aligned}
& E\left[\frac{E_{b}}{I_{0}}\right]_{0} \geq \frac{1}{\frac{\mu N}{G_{p}}+\frac{\mu(N+1)}{2 G_{p}}+\frac{\mu(N+1)}{2 G_{p}} \sum_{j=1}^{J-1}\left(\frac{r_{0}}{r_{j}}\right)^{\alpha} E\left[\phi_{j}\left(\zeta_{j}, \frac{r_{0}}{r_{j}}\right) 10 \zeta_{j} / 10\right]+\frac{\eta}{S}} \\
& \approx \frac{\frac{\mu N}{G_{p}}\left\{3+\sum_{j=1}^{J-1}\left(\frac{r_{0}}{r_{j}}\right)^{\alpha} E\left[\phi_{j}\left(\zeta_{j}, \frac{r_{0}}{r_{j}}\right) 10^{\zeta_{j} / 10}\right]\right\}+\frac{2 \eta}{S}}{2} \\
& E\left[\frac{E_{b}}{I_{0}}\right]_{1} \geq \frac{\frac{1}{2}}{\frac{1}{2} \frac{\mu N}{G_{p}}+\frac{\mu(N+1)}{G_{p}}+\frac{\mu(N+1)}{2 G_{p}} \sum_{j=1}^{J-1}\left(\frac{r_{0}}{r_{j}}\right)^{\alpha} E\left[\phi_{j}\left(\zeta_{j}, \frac{r_{0}}{r_{j}}\right) 10 \varsigma_{j} / 10\right]+\frac{\eta}{S}} \\
& \approx \frac{1}{\frac{\mu N}{G_{p}}\left\{3+\sum_{j=1}^{J-1}\left(\frac{r_{0}}{r_{j}}\right)^{\alpha} E\left[\phi_{j}\left(\zeta_{j}, \frac{r_{0}}{r_{j}}\right) 10 \zeta_{j} / 10\right]\right\}+\frac{2 \eta}{S}} \\
& \begin{aligned}
E\left[\frac{E_{b}}{I_{0}}\right]_{11} & \geq \frac{\frac{1}{2}}{\frac{1}{2} \frac{\mu N}{G_{p}}+\frac{\mu(N+1)}{2 G_{p}}+\frac{\mu(N+1)}{2 G_{p}}\left[2\left(\frac{r_{1}}{r_{0}}\right)^{\alpha}+\sum_{j=2}^{J-1}\left(\frac{r_{1}}{r_{j}}\right)^{\alpha}\right] E\left[\phi_{j}\left(\zeta_{j}, \frac{r_{0}}{r_{j}}\right) 10 \zeta_{j} / 10\right]+\frac{\eta}{S}} \\
& \approx \frac{\frac{\mu N}{G_{p}}\left\{2+\left[2\left(\frac{r_{1}}{r_{0}}\right)^{\alpha}+\sum_{j=2}^{J-1}\left(\frac{r_{1}}{r_{j}}\right)^{\alpha}\right] E\left[\phi_{j}\left(\zeta_{j}, \frac{r_{0}}{r_{j}}\right) 10 \zeta_{j} / 10\right]\right\}+\frac{2 \eta}{S}}{S}
\end{aligned}
\end{aligned}
$$




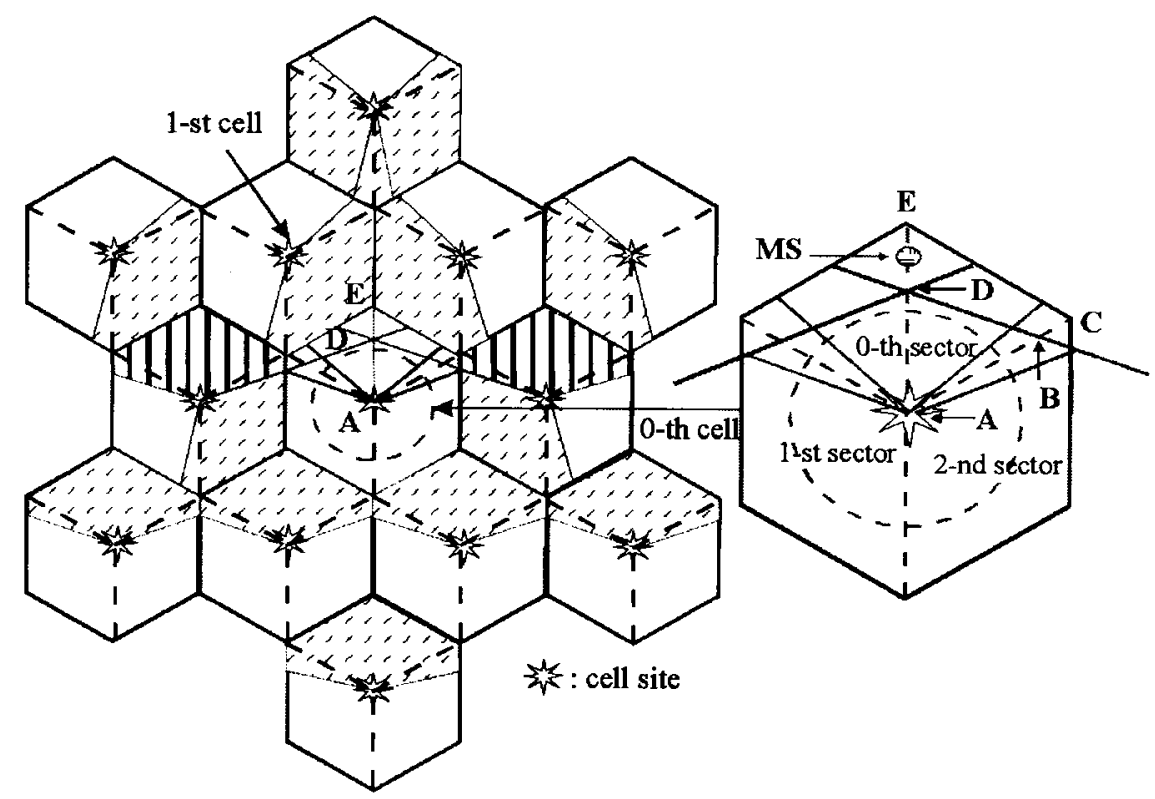

Fig. 11. Interference for mobiles not in the antenna overlapping area. The interfering sectors are shown shaded.

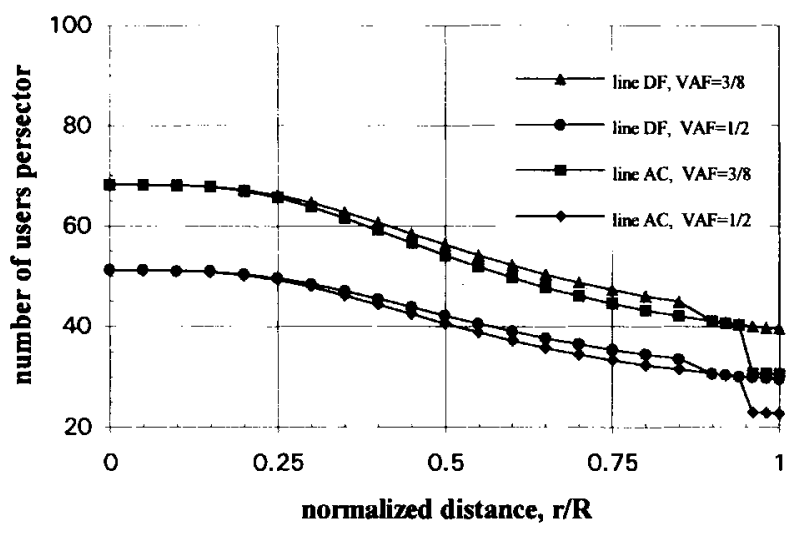

Fig. 12. Forward-link capacity without macrodiversity in sectorized cells.

The radio paths from zeroth BS to mobiles in the softer handoff zone are in a similar environment to the radio paths from the first BS to mobiles in the softer handoff zone. Consequently, the shadow fading and path loss are similar, so the received signal levels at the front end of the mobile's receiver from both the zeroth and first BS are about the same for mobiles in the antenna overlapping area. Because the carrier recovery is coherent for zeroth BS, the signal from zeroth BS is demodulated coherently, while the signal from first BS is not coherently received. From (19), the bit energyto-interference PSD ratio of signals from zeroth BS is shown in (35), given at the bottom of the previous page. When softer handoff is employed for mobiles located in the overlapping areas of antenna patterns, the $E_{b} / I_{0}$ for signals from the first sector BS is shown in (36), given at the bottom of the previous page. Those mobiles located in the soft or softer handoff subzones may be handed off using a soft handoff. When soft handoff is applied, the $E_{b} / I_{0}$ for signals from the first sector BS of a neighboring cell, say the first sector in the first cell, is shown in (37), given at the bottom of the previous page. Notice that the factor of $1 / 2$ in (35)-(37) accounts for the noncoherent demodulation. Also, observe that $2\left(r_{1} / r_{0}\right)^{\alpha}$ in (37) is due to the interference from zeroth sector. When softer handoff is employed, the combined bit energy-to-interference PSD ratio becomes

$$
\frac{E\left[\frac{E_{b}}{I_{0}}\right]_{c}=E\left[\frac{E_{b}}{I_{0}}\right]_{0}+E\left[\frac{E_{b}}{I_{0}}\right]_{1} \geq}{\frac{\mu N}{G_{p}}\left\{3+\sum_{j=1}^{J}\left(\frac{r_{0}}{r_{j}}\right)^{\alpha} E\left[\phi_{j}\left(\zeta_{j}, \frac{r_{0}}{r_{j}}\right) 10 \zeta_{j} / 10\right]\right\}+\frac{2 \eta}{S}} .
$$

$$
\begin{aligned}
E\left[\frac{E_{b}}{I_{0}}\right]_{c} & =E\left[\frac{E_{b}}{I_{0}}\right]_{0}+E\left[\frac{E_{b}}{I_{0}}\right]_{11} \\
& \geq \frac{5+\left[2\left(\frac{r_{1}}{r_{0}}\right)^{\alpha}+\sum_{j=2}^{J-1}\left(\frac{r_{1}}{r_{j}}\right)^{\alpha}\right] E\left[\phi_{j}\left(\zeta_{j}, \frac{r_{0}}{r_{j}}\right) 10^{\zeta_{j} / 10}\right]+\sum_{j=1}^{J-1}\left(\frac{r_{0}}{r_{j}}\right)^{\alpha} E\left[\phi_{j}\left(\zeta_{j}, \frac{r_{0}}{r_{j}}\right) 10^{\zeta_{j} / 10}\right]}{\frac{\mu N}{G_{p}}\left\{2+\left[2\left(\frac{r_{1}}{r_{0}}\right)^{\alpha}+\sum_{j=2}^{J-1}\left(\frac{r_{1}}{r_{j}}\right)^{\alpha}\right] E\left[\phi_{j}\left(\zeta_{j}, \frac{r_{0}}{r_{j}}\right) 10 \zeta_{j} / 10\right]\right\}\left\{3+\sum_{j=1}^{J-1}\left(\frac{r_{0}}{r_{j}}\right)^{\alpha} E\left[\phi_{j}\left(\zeta_{j}, \frac{r_{0}}{r_{j}}\right) 10 \zeta_{j} / 10\right]\right\}}
\end{aligned}
$$




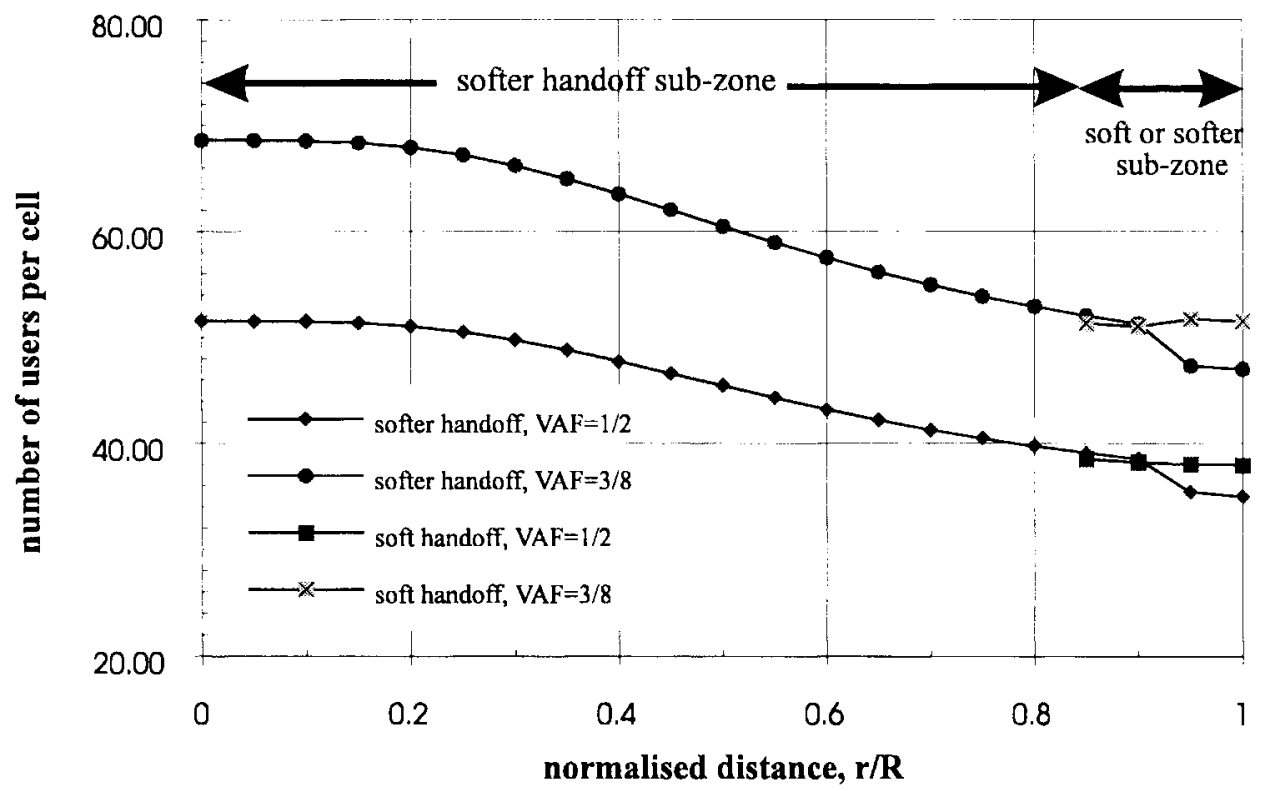

Fig. 13. Forward-link capacity with macrodiversity for mobiles in the antenna pattern overlapping areas (along the ac line).

Similarly, when soft handoff is employed, the combined $E_{b} / I_{0}$ is shown in (39), given at the bottom of the previous page.

For a BER less than $10^{-3}$, the equivalent $\gamma_{\text {req }}$ is $7 \mathrm{~dB}$. According to this specific $\gamma_{\text {req, }}$, the number of users per sector can be evaluated from (38) and (39) as

$$
N \geq \frac{1+\frac{G_{p}}{\mu}\left(\frac{3}{\gamma_{\text {req }}}-\frac{2 \eta}{S}\right)}{3+\sum_{j=1}^{J-1}\left(\frac{r_{0}}{r_{j}}\right)^{\alpha} E\left[\phi_{j}\left(\zeta_{j}, \frac{r_{0}}{r_{j}}\right) 10 \varsigma_{j} / 10\right]}
$$

and as shown in (41), given at the bottom of the page, for the application of softer and soft handoffs, respectively.

The forward-link capacity in terms of the minimal number of users per sector for mobiles in softer handoff and soft or softer handoff subzones can be calculated from (40) and (41). For a CDMA forward-link system having a processing gain of $21 \mathrm{~dB}$ (127 chips per information bit), an $S / \eta$ of $20 \mathrm{~dB}$, and an overlapping angle of $5^{\circ}$, the number of users per sector as a function of normalized distance from $\mathrm{A}$ to $\mathrm{C}$ (along the sector border) are displayed in Fig. 13. When mobiles in the soft or softer subzones employ softer handoff, the capacity in terms of the number of users per sector is improved by macrodiversity from 22 and 30 users per sector to 36 and 47 users per sector for a VAF of $1 / 2$ and $3 / 8$, respectively, while by employing soft handoff, the capacity is improved to 39 and 50 for a VAF of $1 / 2$ and 3/8, respectively. Although there is an increase in interference for mobiles located in the handoff region due to imperfect sectorization, this is overcome by the macrodiversity provided by soft and softer handoffs. Similar to that in unsectorized cells, the forward-link system is limited by mobiles located at $0.84 R$ when soft handoff is not applied. As a consequence, the forward-link capacity is 34 and 46 users per sector for VAF's of $1 / 2$ and $3 / 8$, respectively. The capacity gain due to macrodiversity provided by soft and softer handoffs is 12 and 16 users per sector for a VAF of $1 / 2$ and $3 / 8$, respectively, corresponding to a gain of $53 \%$ compared to that without soft and softer handoffs. As the forward-link capacity loss due to the allocating two traffic channels to a mobile in soft and softer handoffs was $9 \%$, the capacity gain from soft and softer handoffs is $40 \%$ compared to that without soft and softer handoffs. In addition, there is an improvement in communication quality due to the implementation of softer handoff as the system capacity is limited by the mobiles at a distance of $0.84 R$ from their BS.

\section{SECTORIZATION GAIN AND EFFICIENCY FOR IMPERFECT SECTORIZATION}

For a three-sector cell arrangement, the sector antenna pattern has an angle of $120^{\circ}$ for perfect sectorization. On the reverse link, one third of the surrounding areas would be blocked by the antenna pattern, and, hence, the capacity gain would be three times that of the unsectorized cell. Due to the overlapping sector antenna patterns, the interference in sectorized cells is larger than that of sectorized cells having

$$
N \geq \frac{\frac{G_{p}}{\mu \gamma_{\mathrm{req}}}\left\{7+2\left[2\left(\frac{r_{1}}{r_{0}}\right)^{\alpha}+\sum_{j=2}^{J-1}\left(\frac{r_{1}}{r_{j}}\right)^{\alpha}\right] E\left[\phi_{j}\left(\zeta_{j}, \frac{r_{0}}{r_{j}}\right) 10^{\zeta_{j} / 10}\right]+\sum_{j=1}^{J-1}\left(\frac{r_{0}}{r_{j}}\right)^{\alpha} E\left[\phi_{j}\left(\zeta_{j}, \frac{r_{0}}{r_{j}}\right) 10^{\zeta_{j} / 10}\right]\right\}}{\left\{2+\left[2\left(\frac{r_{1}}{r_{0}}\right)^{\alpha}+\sum_{j=2}^{J-1}\left(\frac{r_{1}}{r_{j}}\right)^{\alpha}\right] E\left[\phi_{j}\left(\zeta_{j}, \frac{r_{0}}{r_{j}}\right) 10^{\zeta_{j} / 10}\right]\right\}\left\{3+\sum_{j=1}^{J-1}\left(\frac{r_{0}}{r_{j}}\right)^{\alpha} E\left[\phi_{j}\left(\zeta_{j}, \frac{r_{0}}{r_{j}}\right) 10^{\zeta_{j} / 10}\right]\right\}}
$$




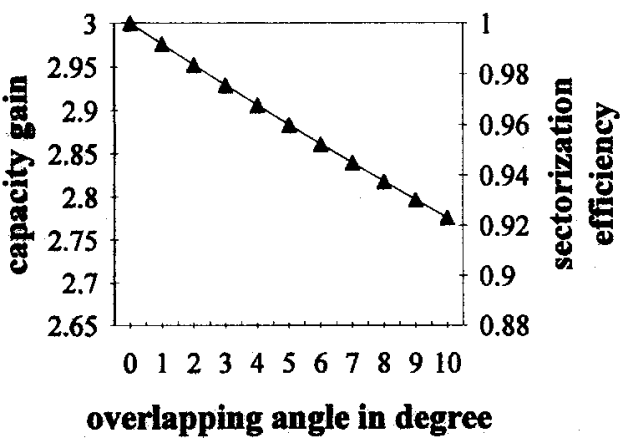

Fig. 14. Reverse-link sectorization gain and efficiency as a function of the overlapping angle $\theta_{o}$.

perfect sectorization. As shown in Fig. 8, the actual sector antenna pattern has an angle that is larger than the nominal angle of $120^{\circ}$. The actual antenna angle is $\left(120+\theta_{\circ}^{\circ}\right)$ rather than $120^{\circ}$. As a result, in a sectorized cell with overlapping angle $\theta_{o}$, only $\left(120+\theta_{o}\right) / 360$ of the interference from the surrounding can be blocked. Therefore, the capacity gain becomes $360 /\left(120+\theta_{0}\right)$ times of the unsectorized cell. We define the efficiency of sectorization as the ratio of the capacity gain with the sector antenna pattern having an overlapping angle $\theta_{o}$ to the nominal antenna pattern of $120^{\circ}$, viz.

$$
\varepsilon_{s}=\frac{\frac{360}{120+\theta_{o}}}{\frac{360}{120}}=\frac{120}{120+\theta_{o}} .
$$

The capacity gain due to sectorization and its corresponding sectorization efficiency for different values of overlapping angles is shown in Fig. 14, where $\theta_{o}=0$ denotes a nominal antenna pattern of $120^{\circ}$. From the graph, the interference on the reverse link increases as the overlapping angle $\theta_{0}$ is increased, which causes the capacity gain and sectorization efficiency to decrease proportionately. For perfect sectorization, the sectorization gain and efficiency are three and one, respectively. For a $\theta_{0}$ of $5^{\circ}$ and $10^{\circ}$, the capacity increase due to sectorization reduces from 3 to 2.88 and 2.77 , respectively, while the corresponding sectorization efficiency is 0.96 and 0.92 for a $\theta_{\circ}$ of $5^{\circ}$ and $10^{\circ}$, respectively.

On the forward link, the capacity for the perfect sectorized sector is 32 and 43 for a VAF of $1 / 2$ and $3 / 8$, respectively, which is the same as that of an unsectorized cell, corresponding to a sectorization efficiency of one. For imperfect sectorization, the interference on the forward link depends on the mobile's location. The interference for mobiles located near the antenna overlapping areas and cell boundaries is higher than that in the central part of sectors. Compared to the capacity of perfect sectorized sectors, without soft and softer handoffs, the forward-link capacity is 22 and 30 users per sector for a VAF of $1 / 2$ and $3 / 8$, respectively, corresponding to a sectorization gain and efficiency of 2.1 and 0.7 , respectively. When soft and softer handoffs are applied, the sectorization gain and efficiency are increased to 2.9 and 0.97 , respectively. Compared with the reverse link, the sectorization efficiency of the forward link is 0.01 times higher than that of the reverse link.

\section{CONCLUSIONS}

The effect of soft and softer handoffs on the system capacity of CDMA systems was analyzed by using an average bit energy-to-interference PSD corresponding to a BER of $10^{-3}$. The capacity in terms of minimal number of users per cell was calculated according to the lower bound of the average bit energy-to-interference PSD. The capacity loss on the forward link due to two traffic channels assigned to a user in the handoff zone was $7.2 \%$, but the capacity gain due to macrodiversity was $7.0 \%$ or $6.1 \%$ for VAF's of $3 / 8$ and $1 / 2$, respectively. There was a small percentage loss of capacity on the forward link. This small percentage of capacity loss did not affect the system capacity, as the forward-link capacity was higher than that of the reverse link due to coherent demodulation. On the other hand, because the system capacity was limited by mobiles at the boundaries between the soft handoff and nonhandoff zones, the excess capacity due to macrodiversity became an improvement in communication quality for mobiles in soft handoff.

For sectorized cells having three sectors per cell, there were overlapping coverage areas between sectors. Consequently, mobiles in these areas were subjected to increase cochannel interference. However, by using soft and softer handoffs, BS's in adjacent sectors supported each mobile by using a forwardlink channel that enabled the mobiles to use maximum ratio combining. As each mobile in the softer handoff process occupied two traffic channels, the capacity loss on forwardlink loss was $9 \%$ for an overlapping angle of $5^{\circ}$. Due to macrodiversity, there was a capacity gain of $40 \%$ irrespective of VAF's compared to that without soft and softer handoffs.

Compared with unsectorized cells, the reverse-link capacity of a system employing three-sector cells with an overlapping angle of $5^{\circ}$ was 2.88 times that of a network employing unsectorized cells, corresponding to a sectorization efficiency of 0.96, irrespective of VAF's. When soft and softer handoffs were applied, the forward-link capacity was 2.9 times that of a network employing unsectorized cells, corresponding to a sectorization efficiency of 0.97 . The sectorization efficiency of the forward link was 0.01 times higher than that of the reverse link. In addition, the application of soft and softer handoffs provided not only a significant improvement on the forwardlink capacity, but also an improvement in communication quality for mobiles near sector boundaries.

\section{REFERENCES}

[1] W. C. Y. Lee, "Overview of cellular, CDMA," IEEE Trans. Veh. Technol., vol. VT-40, no. 2, pp. 291-302, 1991.

[2] C. K. Kwabi, M. P. Mcdonald, L. N. Roberts, W. L. Shanks, N. P. Uhrig, and C. J. Wu, "Operational advantage of the AT\&T CDMA cellular system," in IEEE 42nd VTS Conf., Denver, CO, May 1992, pp. 233-235.

[3] P. Seïté, "Soft handoff in a DS-CDMA microcellular network," in IEEE 44th VTS Conf., Stockholm, Sweden, June 1994, pp. 530-534.

[4] A. J. Viterbi, CDMA: Principle of Spread Spectrum Communication. Reading, MA: Addison-Wesley, 1995.

[5] A. J. Viterbi, A. M. Viterbi, K. S. Gilhousen, and E. Zehavi, "Soft handoff extends CDMA cell coverage and increases reverse link capacity," IEEE J. Select. Areas Commun., vol. 12, no. 8, pp. 1281-1287, 1994.

[6] K. K. Ho, "Architectural design of a code division multiple access cellular system," in IEEE 42nd VTS Conf., Denver, CO, May 1992, pp. 47-49. 
[7] J. Shapira and R. Padovani, "Spatial topology and dynamics in CDMA cellular radio," in IEEE 42nd VTS Conf., Denver, CO, May 1992, pp. 213-216.

[8] G. L. Turin, "The effects of multipath and fading on the performance of direct-sequence CDMA system," IEEE J. Select. Areas Commun., vol. 2, no. 4, pp. 597-603, 1984

[9] K. S. Gilhousen, I. M. Jacobs, R. Padovani, A. J. Viterbi, L. A. Weaver, and C. E. Wheatley, "On the capacity of a cellular CDMA system," IEEE Trans. Veh. Technol., vol. 40, no. 2, pp. 303-311, 1991.

[10] F. Simpson and J. M. Holtzman, "Direct sequence CDMA power control, interleaving, and coding," IEEE J. Select. Areas Commun., vol. 11, no. 7, pp. 1085-1095, 1993.

[11] A. Salmasi and K. S. Gilhousen, "On the system design aspects of code division multiple access (CDMA) applied to digital cellular and personal communications networks," in IEEE 41st VTS Conf., St. Louis, MO, May 1991, pp. 57-62.

[12] J. G. Proakis, Digital Communications. New York: McGraw-Hill, 1983.

[13] W. C. Jakes, Microwave Mobile Radio Communications. New York: IEEE Press, 1974.

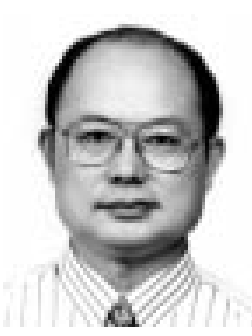

Chin-Chun Lee (S'94-M'95) was born in Kimmen, R.O.C., on October 17, 1953. He received the B.Sc. and M.Sc. degrees in electrical engineering from Chung Cheng Institute of Technology, Taiwan, R.O.C., in 1976 and 1984, respectively, and the $\mathrm{Ph} . \mathrm{D}$. degree in electronic engineering from the University of Southampton, Southampton, U.K., in 1995.

He was with the R.O.C. Army Signal School from 1976 to 1982 , where he lectured on tactical multiplexers and radio transceivers for army technicians. He joined the Department of Electrical Engineering, Chung Cheng Institute of Technology, as a Lecturer in 1984, became an Associate Professor in 1995, and remained in the department until 1998. He is currently with the Department of Communication and Computer Technology, Ta-Yeh University, Changhwa, Taiwan. His research interests include cellular and personal communication systems, capacity evaluation, power control, handoff, and rain-fade compensation in satellite communications.

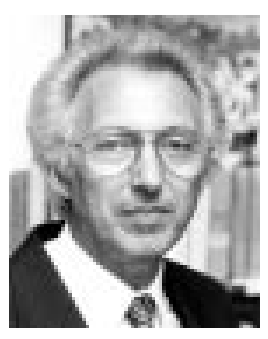

Raymond Steele (SM'80-F'96) received the B.Sc. degree in electrical engineering from Durham University, U.K., in 1959 and the Ph.D. and D.Sc degrees from the Loughborough University of Technology, U.K., in 1975 and 1983, respectively.

$\mathrm{He}$ is the Chairman of Multiple Access Communications Ltd. and Head of the Communications Research Group in the Department of Electronics and Computer Science, Southampton University, Southampton, U.K. Before receiving the B.Sc. degree, he was an indentured Apprentice Radio Engineer. After research and development posts with E K Cole, Cossor Radar and Electronics, and Marconi, he joined the lecturing staff at the Royal Naval College, London, U.K. He moved to Loughborough University in 1968, where he lectured and directed a research group in digital encoding of speech and picture signals. During the summers of 1975, 1977, and 1978, he was a Consultant to the Acoustics Research Department at Bell Laboratories. In 1979, he joined the Communications Methods Research Department, Crawford Hill Laboratory, Bell Laboratories, NJ. He has been a Professor of Communications in the Department of Electronics and Computer Science, University of Southampton, since 1983. From 1983 to 1986, he was a Nonexecutive Director of Plessey Research and Technology and from 1983 to 1989 a Consultant to British Telecom Research Laboratories. In 1986, he formed Multiple Access Communications Ltd., a company concerned with digital mobile radio systems. He is the author of Delta Modulation Systems (Halsted, NY: 1975), Editor of Mobile Radio Communications (New York: IEEE Press and Wiley, 1992), and coauthor of Source-Matched Mobile Communications (New York: IEEE Press and Wiley, 1995). He is also the author of over 200 technical publications.

Dr. Steele and his coauthors were awarded the Marconi Premium in 1979 and in 1989 and the Bell System Technical Journal's Best Mathematics, Communications, Techniques, Computing and Software, and Social Sciences Paper in 1981. He has been a conference and session organizer of numerous international conferences and a keynote speaker at many international meetings. $\mathrm{He}$ is also a Senior Technical Editor of the IEEE COMMUNICATIONS MAGAZINE and a Member of the Advisory Boards of IEEE Personal Communications (the magazine of nomadic communications and computing) and the International Journal of Wireless Information Networks. He is a Fellow of the Royal Academy of Engineering and IEE and a Member of the IEEE Avant Garde. 